\title{
Responding to Clinicians Who Fail to Follow Patient Safety Practices: Perceptions of Physicians, Nurses, Trainees, and Patients
}

\author{
Todd H. Driver, BA ${ }^{1}$, Patricia P. Katz, $\mathrm{PhD}^{2}$, Laura Trupin, $\mathrm{MPH}^{2}$, Robert M. Wachter, $\mathrm{MD}^{2 *}$
}

${ }^{1}$ School of Medicine, University of California, San Francisco, San Francisco, California; ${ }^{2}$ Department of Internal Medicine, University of California, San Francisco, California.

BACKGROUND: The field of patient safety grapples with the tension between a "no-blame" systems approach and the need to hold providers accountable for substandard performance. Attitudes of clinicians and patients regarding methods of promoting adherence to safety practices have not been described.

METHODS: We surveyed attending and resident physicians in the University of California, San Francisco (UCSF) Department of Medicine, nurses and inpatients at the UCSF Medical Center, and UCSF medical students regarding attitudes toward violations of 3 safety protocols: hand hygiene, fall risk assessment, and preoperative time-out.

RESULTS: Responses to protocol lapses were grouped into 3 categories: feedback (universally endorsed and thus excluded from the analysis), public reporting, and penalty (fines, suspension, firing). We examined group differences regarding whether public reporting and penalties were ever appropriate and the number of transgressions at which public reporting and penalties were favored. Respondents favored both public reporting and penalties more frequently for not conducting a preoperative time-out or fall risk assessment than for hand-hygiene lapses (public reporting: time-out, odds ratio [OR]: 2.82 [95\% confidence interval \{Cl\}: 2.03-3.91]; fall, OR: 1.47 [95\% Cl: 1.09-1.98]. Penalty: time-out, OR: 4.29 [95\% Cl: 2.97-6.20]; fall, OR: 1.74 [95\% $\mathrm{Cl}: 1.27-2.37])$. Penalties were endorsed more frequently than public reporting for all groups and scenarios. Attending physicians and patients expressed similar attitudes regarding public reporting and penalties, but patients favored penalties after significantly fewer transgressions $(P<0.05)$.

CONCLUSION: After a decade emphasizing no-blame responses to patient safety hazards, both healthcare providers and patients now believe clinicians should be held accountable for following basic safety protocols. Journal of Hospital Medicine 2014;9:99-105. (C) 2013 Society of Hospital Medicine
Healthcare delivery organizations are under increasing pressure to improve patient safety. The fundamental underpinning of efforts to improve safety has been the establishment of a "no-blame" culture, one that focuses less on individual transgressions and more on system improvement. ${ }^{1,2}$ As evidence-based practices to improve care have emerged, and the pressures to deliver tangible improvements in safety and quality have grown, providers, healthcare system leaders, and policymakers are struggling with how best to balance the need for accountability with this no-blame paradigm.

In dealing with areas such as hand hygiene, where there is strong evidence for the value of the practice yet relatively poor adherence in many institutions, Wachter and Pronovost have argued that the scales need to tip more in the direction of accountability, including the imposition of penalties for clinicians

*Address for correspondence and reprint requests: Robert $\mathrm{M}$. Wachter, MD, Department of Medicine, Room M-994, 505 Parnassus Avenue, San Francisco, CA 94143-0120; Telephone: 415-476-5632; Fax: 415-502-5869; E-mail: bobw@medicine.ucsf.edu

Additional Supporting Information may be found in the online version of this article.

Received: September 25, 2013; Revised: November 20, 2013; Accepted: November 24, 2013

2013 Society of Hospital Medicine DOI 10.1002/jhm.2136

Published online in Wiley Online Library (Wileyonlinelibrary.com). who habitually fail to follow certain safety practices. ${ }^{3}$ Although not obviating the critical importance of systems improvement, they argue that a failure to enforce such measures undermines trust in the system and invites external regulation. Chassin and colleagues made a similar point in arguing for the identification of certain "accountability measures" that could be used in public reporting and pay-for-performance programs. ${ }^{4}$

Few organizations have enacted robust systems to hold providers responsible for adhering to accountability measures. ${ }^{4}$ Although many hospitals have policies to suspend clinical privileges for failing to sign discharge summaries or obtain a yearly purified protein derivative test, few have formal programs to identify and deal with clinicians whose behavior is persistently problematic. ${ }^{3}$ Furthermore, existing modes of physician accountability, such as state licensing boards, only discipline physicians retroactively (and rarely) when healthcare organizations report poor performance. State boards typically do not consider prevention of injury, such as adherence to safety practices, to be part of their responsibility. ${ }^{5}$ Similarly, credentialing boards (eg, the American Board of Internal Medicine) do not assess adherence to such practices in coming to their decisions.

It is estimated that strict adherence to infection control practices, such as hand hygiene, could prevent 
over 100,000 hospital deaths every year; adherence to other evidence-based safety practices such as the use of a preoperative "time-out" would likely prevent many more deaths and cases of medical injury., Although there are practical issues, such as how to audit individual clinician adherence in ways that are feasible and fair, that make enforcing individual provider accountability challenging, there seems little doubt that attitudes regarding the appropriateness of enacting penalties for safety transgressions will be key determinants of whether such measures are considered. Yet no study to date has assessed the opinions of different stakeholders (physicians, nurses, trainees, patients) regarding various strategies, including public reporting and penalties, to improve adherence to safety practices. We aimed to assess these attitudes across a variety of such stakeholders.

\section{METHODS}

\section{Survey Development and Characteristics}

To understand the perceptions of measures designed to improve patient safety, we designed a survey of patients, nurses, medical students, resident physicians, and attending physicians to be administered at hospitals associated with the University of California, San Francisco (UCSF). Institutional review board approval was obtained from the UCSF Committee on Human Research, and all respondents provided informed consent.

The survey was developed by the authors and pilot tested with 2 populations. First, the survey was administered to a group of 12 UCSF Division of Hospital Medicine research faculty; their feedback was used to revise the survey. Second, the survey was administered to a convenience sample of 2 UCSF medical students, and their feedback was used to further refine the survey.

The questionnaire presented 3 scenarios in which a healthcare provider committed a patient-safety protocol lapse; participants were asked their opinions about the appropriate responses to each of the violations. The 3 scenarios were: (1) a healthcare provider not properly conducting hand hygiene before a patient encounter, (2) a healthcare provider not properly conducting a fall risk assessment on a hospitalized patient, and (3) a healthcare provider not properly conducting a preoperative timeout prior to surgery. For each scenario, a series of questions was asked about a variety of institutional responses toward a provider who did not adhere to each safety protocol. Potential responses included feedback (email feedback, verbal feedback, meeting with a supervisor, a quarterly performance review meeting, and a quarterly report card seen only by the provider), public reporting (posting the provider's infractions on a public website), and penalties (fines, suspension without pay, and firing).

We chose the 3 practices because they are backed by strong evidence, are relatively easy to perform, are inexpensive, are linked to important and common harms, and are generally supported within the patientsafety community. Improved adherence to hand hygiene significantly reduces infection transmission in healthcare settings. ${ }^{7-11}$ Performing fall risk assessments has been shown to reduce falls in hospitalized patients, ${ }^{12}$ and using preoperative checklists, including a surgical time-out, can reduce mortality and complication risks by approximately $40 \% .^{13}$

Respondents were asked how many cases of documented nonadherence would be necessary for the penalties to be appropriate (1 time, 2-5 times, 6-10 times, 11-15 times, 16+ times, or would never be appropriate). Finally, respondents were asked to rate the potential harm to patients of each protocol lapse (none-low, medium, or high).

Demographic information collected from the healthcare providers and medical students included age, gender, position, department, and years' experience in their current position. Demographic information collected from the patients included age, gender, insurance status, race, education level, household income level, and relationship status.

\section{Survey Administration}

Surveys were administered to convenience samples of 5 groups of individuals: attending physicians in the UCSF Department of Internal Medicine based at UCSF Medical Center and the San Francisco Veterans Affairs Medical Center, nurses at UCSF Medical Center, residents in the UCSF internal medicine residency program, medical students at UCSF, and inpatients in the internal medicine service at UCSF Medical Center's Moffitt-Long Hospital. Attending physicians and nurses were surveyed at their respective departmental meetings. For resident physicians and medical students, surveys were distributed at the beginning of lectures and collected at the end.

Patients were eligible to participate if they spoke English and were noted to be alert and oriented to person, time, and place. A survey administrator located eligible patients in the internal medicine service via the electronic medical record system, determined if they were alert and oriented, and approached each patient in his or her room. If the patients verbally consented to consider participation, the surveys were given to them and retrieved after approximately 30 minutes.

Healthcare professionals were offered the opportunity to enter their e-mail addresses at the end of the survey to become eligible for a drawing for a $\$ 100$ gift card, but were informed that their e-mail addresses would not be included in the analytic dataset. Inpatients were not offered any incentives to participate. All surveys were administered by a survey monitor in paper form between May 2011 and July 2012. 
TABLE 1. Characteristics of Survey Respondents

\begin{tabular}{lccccc}
\hline & $\begin{array}{c}\text { Attending } \\
\text { Physician }\end{array}$ & $\begin{array}{c}\text { Resident } \\
\text { Physician }\end{array}$ & Nurse & $\begin{array}{c}\text { Medical } \\
\text { Student }\end{array}$ & Patient \\
\hline No. & 22 & 33 & 61 & 47 & 20 \\
Response rate $^{*}$ & $73 \%$ & $73 \%$ & $59 \%$ & $54 \%$ & $87 \%$ \\
Age, y, mean \pm SD & $36 \pm 5$ & $28 \pm 2$ & $44 \pm 11$ & $27 \pm 3$ & $48 \pm 16$ \\
Sex, female, \% (n) & $32 \%(7)$ & $67 \%(22)$ & $88 \%(53)$ & $66 \%(31)$ & $47 \%(9)$ \\
\hline
\end{tabular}

NOTE: Abbreviations: SD, standard deviation.

*The denominator for response rate was defined as those who received the survey.

\section{Data Analysis}

Data analysis was conducted using the Statistical Analysis Software (SAS) package (SAS Institute Inc., Cary, NC) and Stata (StataCorp, College Station, TX). Descriptive analysis and frequency distributions were tallied for all responses. Responses to protocol lapses were grouped into 3 categories: feedback, public reporting, and penalty as described above. As all surveyed groups endorsed feedback as an appropriate response to all of the scenarios, we did not examine feedback, concentrating our analysis instead on public reporting and penalties.

Appropriateness ratings for each response to each protocol lapse were aggregated in 2 ways: ever appropriate (ie, the response would be appropriate after some number of documented lapses) versus never appropriate, and the threshold for the response. Whereas public reporting was only asked about as a single option, 3 separate responses were collapsed into the single response, "penalties": fine, suspension, or firing. Individuals were classified as endorsing a penalty if they rated any 1 of these responses as ever appropriate. The threshold for penalty was the smallest number of occurrences at which 1 of the penalty responses was endorsed.

Differences among the 5 groups in the perceived harm of each protocol lapse were tested with $\chi^{2}$ analyses. Group differences in ratings of whether public reporting and penalties were ever appropriate were tested with logistic regression analyses for each scenario separately, controlling for age, sex, and perceived harm of the protocol lapse. To determine if the 5 groups differed in their tendency to support public reporting or penalties regardless of the type of protocol lapse, we conducted logistic regression analyses across all 3 scenarios, accounting for multiple observations per individual through use of clustercorrelated robust variance. ${ }^{14}$ Differences among groups in the number of transgressions at which public reporting and penalties were supported were examined with log-rank tests.

\section{RESULTS}

A total of 287 individuals were given surveys, and 183 completed them: 22 attending physicians, 33 resident physicians, 61 nurses, 47 medical students, and
20 patients (overall response rate 64\%). Response rate for attending and resident physicians was $73 \%$, for nurses 59\%, and for medical students $54 \%$. Among patients who were approached and agreed to accept a survey, $87 \%$ returned completed surveys (Table 1). The average age of attending physicians was 35.8 years (standard deviation [SD]: 5.3), residents was 28.3 years (SD: 1.7 ), nurses was 43.6 years (SD: 11.1), medical students was 26.6 years (SD: 2.9), and inpatients was 48.2 years (SD: 15.9). Thirty-two percent of attending physicians were female, $67 \%$ of resident physicians were female, $88 \%$ of nurses were female, $66 \%$ of medical students were female, and $47 \%$ of inpatients were female.

\section{Perceived Harm}

Out of the 3 scenarios presented in in the survey, participants believed that not conducting preoperative time-outs in surgery presented the highest risk to patient safety, with $57 \%$ (residents) to $86 \%$ (nurses) rating the potential harm as high (Figure 1). Not conducting fall risk assessments was perceived as second most potentially harmful, and not properly practicing hand hygiene was perceived as least potentially harmful to patient safety. There were significant differences among groups in perceptions of potential harm for all 3 scenarios $(P<0.001$ for all $)$.

\section{Appropriateness of Public Reporting and Penalties}

Public reporting was viewed as ever appropriate by $34 \%$ of all respondents for hand-hygiene protocol lapses, $58 \%$ for surgical time-out lapses, and $43 \%$ for fall risk assessment lapses. There were no significant differences among groups in endorsement of public reporting for individual scenarios (Figure 2). Penalties were endorsed more frequently than public reporting for all groups and all scenarios. The proportion of attending physicians and patients who rated penalties as ever appropriate were similar for each scenario. Residents, medical students, and nurses were less likely than patients and attending physicians to support penalties $(P<0.05$ for all differences).

The aggregated analysis revealed that nurses and medical students were significantly less likely than patients to endorse public reporting across scenarios. In terms of endorsement of penalties, we found no significant differences between attending physicians and patients, but residents (odds ratio [OR]: 0.09, 95\% confidence interval [CI]: 0.03-0.32), students (OR: 0.12, 95\% CI: 0.04-0.34), and nurses (OR: 0.17, 95\% CI: 0.03-0.41) had significantly lower odds of favoring penalties than did patients (Table 2).

Across all surveyed groups, public reporting was more often supported for lapses of surgical timeout (OR: 2.82, 95\% CI: 2.03-3.91) and fall risk assessment protocols (OR: 1.47, 95\% CI: 1.09-1.98) than for the referent, hand-hygiene lapses. Across all groups, penalties were more likely to be supported for 
Perceived Potential to Harm Patients: Hand Hygiene

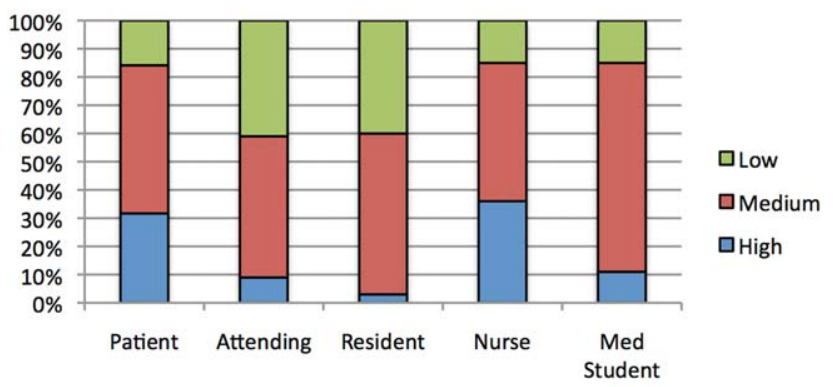

Perceived Potential to Harm Patients: Surgical Timeout

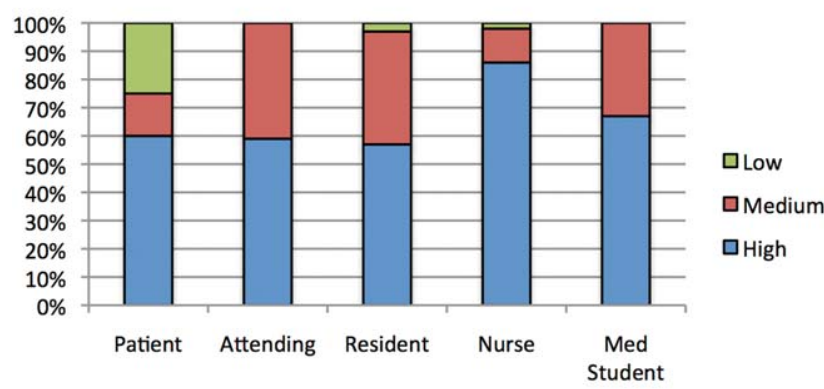

Perceived Potential to Harm Patients: Fall Assessment

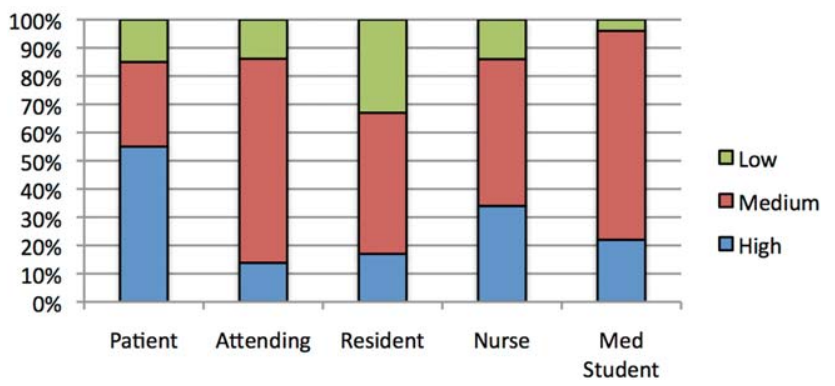

FIG. 1. Ratings by health professionals and patients of potential harm from safety lapses. Blue bars denote high perceived risk, whereas red bars and green bars denote medium and low perceived risks, respectively, of each safety protocol transgression scenario.

surgical timeout (OR: 4.29, 95\% CI: 2.97-6.20) and fall risk assessment protocol lapses (OR: 1.74, 95\% CI: 1.27-2.37) than for hand-hygiene lapses.

\section{Thresholds for Public Reporting and Penalties}

The log-rank test showed no significant differences among the surveyed groups in the number of transgressions at which public reporting was deemed appropriate in any of the 3 scenarios $(P=0.37, P=0.71$, and $P=0.32$ for hand hygiene, surgical time-out, and fall risk assessment, respectively) (Figure 3). However, patients endorsed penalties after significantly fewer occurrences than residents, medical students, and nurses for all 3 scenarios $(P<0.001$ for all differences), and at a significantly lower threshold than attending physicians for surgical timeout and fall risk assessment $(P<0.001$ and $P=0.03$, respectively).

\section{DISCUSSION}

This survey assessed attitudes of healthcare professionals, trainees, and inpatients toward public reporting and penalties when clinicians do not follow basic safety protocols. Respondents tended to favor more aggressive measures when they deemed the safety risk from protocol violations to be higher. Almost all participants favored providing feedback after safety protocol lapses. Healthcare professionals tended to favor punitive measures, such as fines, suspension, and firing, more than public reporting of transgressions. Patients had a lower threshold than both providers and trainees for public reporting and punitive measures. In aggregate, our study suggests that after a decade of emphasis on a no-blame response to patient safety hazards, both healthcare providers and patients now believe clinicians should be held accountable for following basic safety protocols, though their thresholds and triggers vary.

A surprising finding was that providers were more likely to favor penalties (such as fines, suspension, or firing) than public reporting of safety transgressions. Multiple studies have suggested that public reporting of hospital quality data has improved adherence to care processes and may improve patient outcomes. ${ }^{15-17}$ Although our data do not tell us why clinicians appear to be more worried about public reporting than penalties, they do help explain why transparency has been a relatively powerful strategy to motivate changes in practice, even when it is unaccompanied by significant shifts in consumer choices. ${ }^{18}$ It would be natural to consider public reporting to be a "softer" strategy than fines, suspension, or firing; however, our results indicate that many clinicians do not see it that way. Alternatively, the results could also suggest that clinicians prefer measures that provide more immediate feedback than public reporting generally provides. These attitudes should be considered when enacting public reporting strategies.

Another interesting finding was that patients and attending physicians tended to track together regarding their attitudes toward penalties for safety lapses. Although patients had a lower threshold for favoring penalties than attendings, similar proportions of patients and attending physicians believed that penalties should be enacted for safety transgressions, and both groups were more penal than physician trainees and nurses. We speculate that attendings and patients may have the most "skin in the game," patients as the ones directly harmed by a preventable adverse event, and attending physicians as the most responsible clinicians, at least in the eyes of the malpractice system, licensing boards, and credentials committees.

Even though our study illustrates relatively high levels of endorsement for aggressive measures to deal with clinicians who fail to follow evidence-based safety practices, a shift in this direction has risks and 


\section{Hand hygiene}

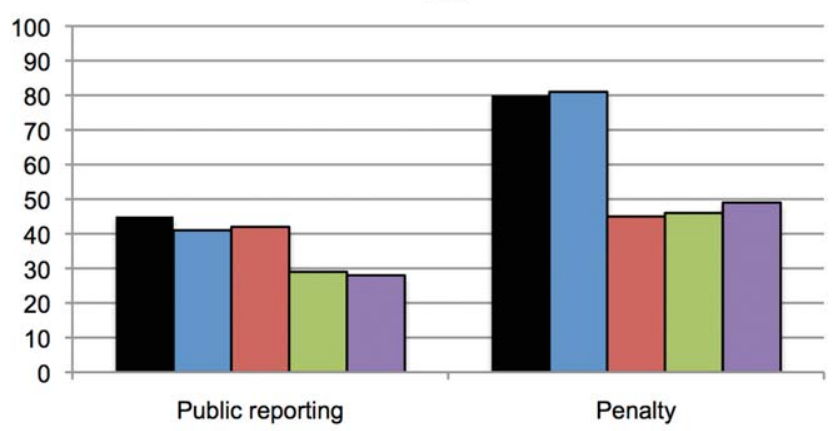

\section{Surgical timeout}

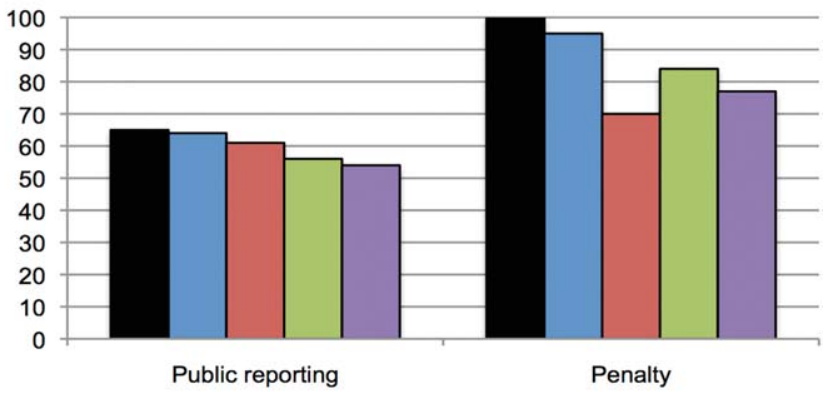

Fall assessment

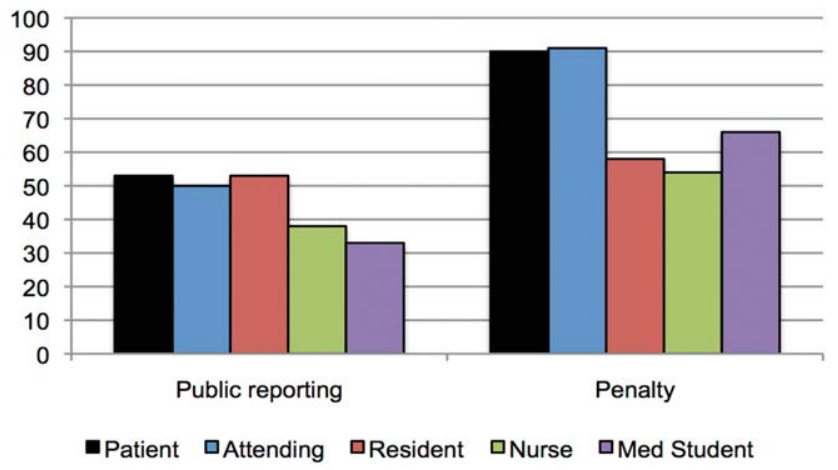

FIG. 2. Percent of health professionals and patients who rated public reporting and penalty as ever appropriate. Each bar represents the percent of attending physicians, resident physicians, nurses, medical students, or inpatients who rated public reporting and penalty as ever appropriate (after some number of transgressions) for each safety protocol scenario.

benefits. The no-blame paradigm in patient safety grew out of a need to encourage open discussion about medical mistakes. ${ }^{2}$ Whereas shifting away from a purely no- blame approach may lead to greater adherence with safety practices, and one hopes fewer cases of preventable harm, it also risks stifling the open discussions about medical errors that characterize learning organizations. ${ }^{13,19}$ Because of this, a movement in this direction should be undertaken carefully, starting first with a small number of wellestablished safety practices, and ensuring that robust education and system improvements precede and accompany the imposition of penalties for nonadherence.

Our study has limitations. The survey was developed using convenience samples of UCSF faculty and medical
TABLE 2. Likelihood of Endorsing Public Reporting or Penalties at Any Time by Group and Scenario

\begin{tabular}{lcc}
\hline & \multicolumn{2}{c}{ Odds Ratio $(95 \% \mathrm{Cl})$} \\
\cline { 2 - 3 } & Public Reporting & Penalty \\
\hline Group, across all scenarios & Reference & Reference \\
Patients & $0.58(0.17-2.01)$ & $0.88(0.20-3.84)$ \\
Attending physicians & $0.42(0.12-1.52)$ & $0.09(0.02-0.32)$ \\
Resident physicians & $0.32(0.12-0.88)$ & $0.17(0.03-0.41)$ \\
Nurses & $0.22(0.06-0.80)$ & $0.12(0.04-0.34)$ \\
Medical students & & \\
Scenario, across all groups & Reference & Reference \\
Hand hygiene & $2.82(2.03-3.91)$ & $4.29(2.97-6.20)$ \\
Surgical time-out & $1.47(1.09-1.98)$ & $1.74(1.27-2.37)$ \\
Fall assessment & & \\
\hline
\end{tabular}

NOTE: Odds ratios and proportions were derived from logistic regression models including group, scenario, age, and sex adjusting for clustering within individuals. Abbreviations: $\mathrm{Cl}$, confidence interval.

students, so broader inclusion of physicians, nurses, trainees, and patients may have yielded a different survey instrument. As a survey, we cannot be certain that any of the groups' responses in real life (eg, in a vote of the medical staff on a given policy) would mirror their survey response. Additionally, the responses to protocol lapses did not include all possible administrative responses, such as mandatory training/remediation or rewards for positive behaviors. The responses could have also been different if participants were presented with different patient safety scenarios. The study population was limited in several ways. Attending and resident physicians were drawn from an academic department of internal medicine; it is possible that other specialties would have different attitudes. Patients were relatively young (likely due to the inclusion criteria), as were attending physicians (due to oversampling of hospitalist physicians). The relatively small number of participants could also limit statistical power to detect differences among groups. Additionally, the study population was limited to patients and healthcare professionals in academic medical centers in San Francisco. It is possible that attitudes would be different in other regions and practice settings.

The no-blame approach to patient safety has been crucial in refocusing the lens on systems failures and in encouraging the active engagement by clinicians, particularly physicians. ${ }^{2,3}$ On the other hand, there are legitimate concerns that a unidimensional noblame approach has permitted, perhaps even promoted, nonadherence to evidence-based safety practices that could prevent many cases of harm. Although it may not be surprising that patients favor harsher consequences for providers who do not follow basic safety protocols, our study demonstrates relatively widespread support for such consequences even among clinicians and trainees. However, all groups appear to recognize the nuances underlying this set of issues, with varying levels of enthusiasm for punitive responses based on perceived risk and number of 


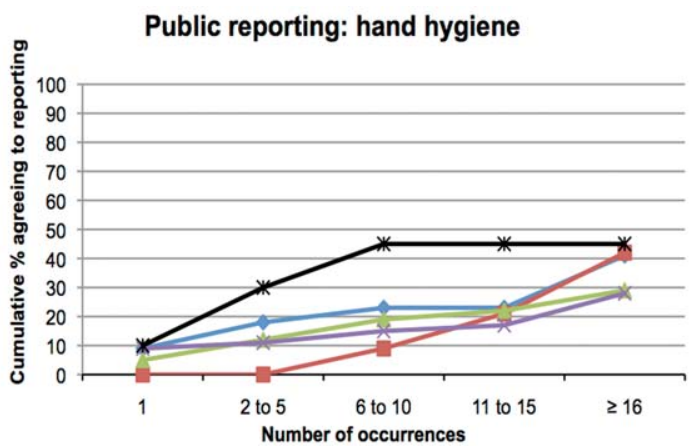

Public reporting: surgical timeout

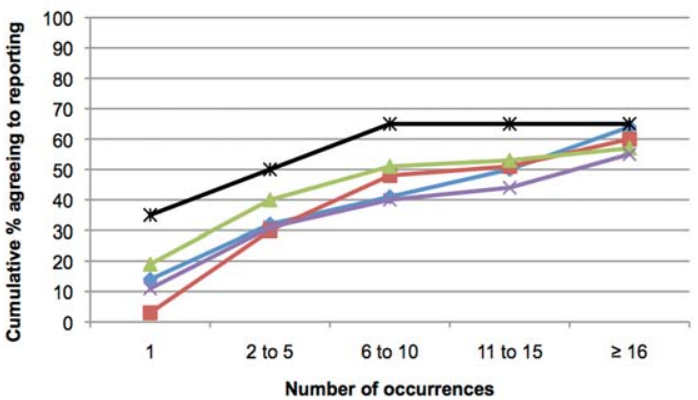

Public reporting: fall assessment

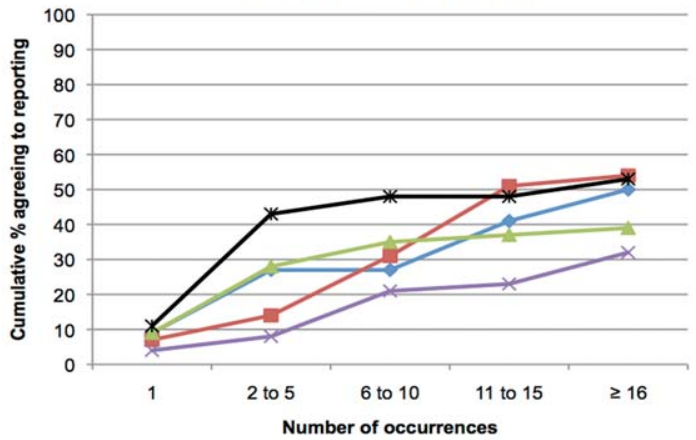

Penalty: hand hygiene

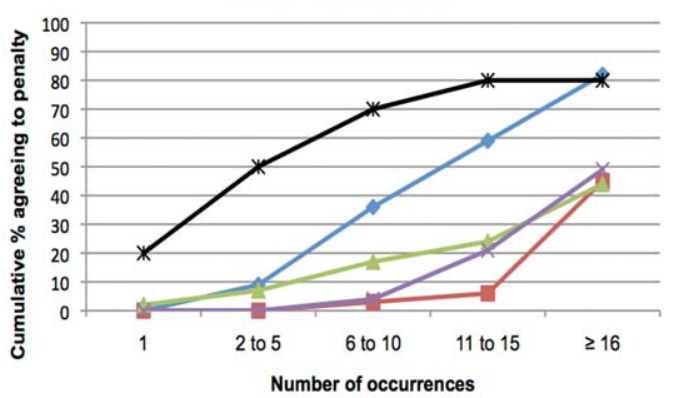

Penalty: surgical timeout

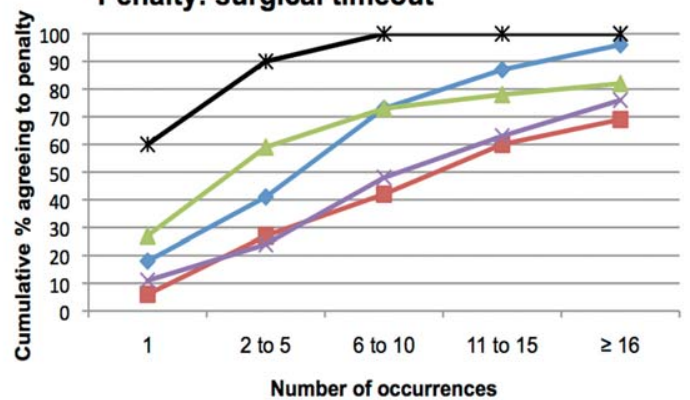

Penalty: fall assessment

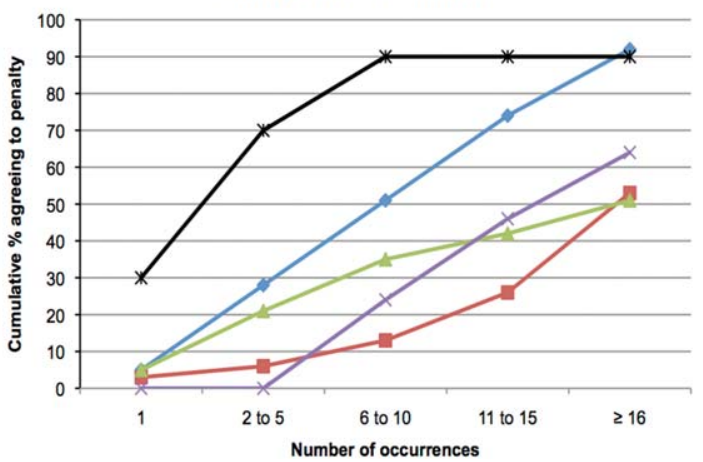

FIG. 3. Thresholds for public reporting and penalty for health professionals and patients by scenario. Number of occurrences is the number of failures to perform a given safety practice before the respondent favored the action. For example, $20 \%$ of patients favored 1 type of penalty (fine, suspension, or firing) after 1 documented episode of a clinician's failure to clean his or her hands; $80 \%$ of patients favored a penalty after 11 to 15 documented transgressions.

transgressions. Future studies are needed to investigate how best to implement public reporting and penalties in ways that can maximize the patient safety benefits.

\section{Acknowledgements}

The authors are grateful to the clinicians, trainees, and patients who participated in the survey.

\section{References}

1. Wachter RM. Understanding Patient Safety. 2nd ed. New York, NY: McGraw Hill Medical; 2012.

2. Leape LL. Error in medicine. JAMA. 1994;272(23):1851-1857.

3. Wachter RM, Pronovost PJ. Balancing "no blame" with accountability in patient safety. N Engl J Med. 2009;361(14):1401-1406.

4. Chassin MR, Loeb JM, Schmaltz SP, Wachter RM. Accountability measures—using measurement to promote quality improvement. $N$ Engl J Med. 2010;363(7):683-688.

5. Leape LL, Fromson JA. Problem doctors: is there a system-level solution? Ann Intern Med. 2006;144(2):107-115.

6. Haynes AB, Weiser TG, Berry WR, et al. A surgical safety checklist to reduce morbidity and mortality in a global population. N Engl J Med. 2009;360(5):491-499.

7. Schweon SJ, Edmonds SL, Kirk J, Rowland DY, Acosta C. Effectiveness of a comprehensive hand hygiene program for reduction of infec- tion rates in a long-term care facility. Am I Infect Control. 2013; 41(1):39-44.

8. Ling ML, How KB. Impact of a hospital-wide hand hygiene promotion strategy on healthcare-associated infections. Antimicrob Resist Infect Control. 2012;1(1):13.

9. Alsubaie S, Maither AB, Alalmaei W, et al. Determinants of hand hygiene noncompliance in intensive care units. Am J Infect Control. 2013;41(2):131-135

10. Kirkland KB, Homa KA, Lasky RA, Ptak JA, Taylor EA, Splaine ME. Impact of a hospital-wide hand hygiene initiative on healthcareassociated infections: results of an interrupted time series. BMJ Qual Saf. 2012;21(12):1019-1026.

11. Ho ML, Seto WH, Wong LC, Wong TY. Effectiveness of multifaceted hand hygiene interventions in long-term care facilities in Hong Kong: a cluster-randomized controlled trial. Infect Control Hosp Epidemiol. 2012;33(8):761-767.

12. Neiman J, Rannie M, Thrasher J, Terry K, Kahn MG. Development, implementation, and evaluation of a comprehensive fall risk program. J Spec Pediatr Nurs 2011;16(2):130-139.

13. Borchard A, Schwappach DL, Barbir A, Bezzola P. A systematic review of the effectiveness, compliance, and critical factors for implementation of safety checklists in surgery. Ann Surg. 2012;256(6):925-933.

14. Williams RL. A note on robust variance estimation for clustercorrelated data. Biometrics. 2000;56(2):645-646.

15. Lindenauer PK, Remus D, Roman S, et al. Public reporting and pay for performance in hospital quality improvement. N Engl I Med. 2007;356(5):486-496. 
16. Hannan EL, Kilburn H Jr, Racz M, Shields E, Chassin MR. Improving the outcomes of coronary artery bypass surgery in New York State. JAMA. 1994;271(10):761-766.

17. Rosenthal GE, Quinn L, Harper DL. Declines in hospital mortality associated with a regional initiative to measure hospital performance. Am J Med Qual. 1997;12(2):103-112.
18. Marshall MN, Shekelle PG, Leatherman S, Brook RH. The public release of performance data: what do we expect to gain? A review of the evidence. JAMA. 2000;283(14):1866-1874.

19. Eisenberg JM. Continuing education meets the learning organization: the challenge of a systems approach to patient safety. J Contin Educ Health Prof. 2000;20(4):197-207. 